\title{
Virtual Bronchoscopic Navigation for Peripheral Pulmonary Lesions
}

\author{
Fumihiro Asano ${ }^{a}$ b Ralf Eberhardt ${ }^{b}$ Felix J.F. Herth ${ }^{b}$

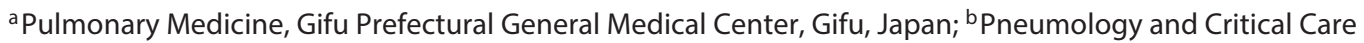 \\ Medicine, Thoraxklinik, University of Heidelberg, Heidelberg, Germany
}

\section{Key Words}

Bronchoscopy · Endobronchial ultrasound · Lung cancer ·

Navigational bronchoscopy · Peripheral pulmonary lesions .

Solitary pulmonary nodule - Transbronchial biopsy .

Ultrathin bronchoscope - Virtual bronchoscopy

\begin{abstract}
Virtual bronchoscopic navigation (VBN) is a method in which the bronchoscope is guided on the bronchial route to a peripheral lesion using virtual bronchoscopic images. In reports on VBN for peripheral pulmonary lesions searched in PubMed as of November 2013, the diagnostic yield by ultrathin bronchoscopy in combination with computed tomography and VBN was within the range of $65.4-81.6 \%$. Using endobronchial ultrasonography with a guide sheath (EBUSGS) and VBN, it was between 63.3 and $84.4 \%$, and using X-ray fluoroscopy and VBN, it was between 62.5 and $78.7 \%$. The overall diagnostic yield was $73.8 \%$ [95\% confidence interval (CI) $70.9-76.8 \%]$ and that for lesions $\leq 2 \mathrm{~cm}$ was $67.4 \%$ (95\% $\mathrm{Cl}$ 63.3-71.5\%). These values indicate high diagnostic rates. In randomized comparative trials, the combination of VBN with EBUS-GS improved the diagnostic yield and shortened the examination time. The diagnostic yields for lesions in the right upper lobe, those invisible on posterior-anterior radiographs and those located in the peripheral third of the lung
\end{abstract}

field were improved by VBN on ultrathin bronchoscopy in combination with X-ray fluoroscopy. The usefulness of VBN was also found on meta-analysis. Taken together, VBN is a promising navigational bronchoscopy method as it requires no specific training, has a low overall complication rate of $1.0 \%(95 \% \mathrm{Cl} 0.2-1.8 \%)$ and does not directly induce or cause severe complications. To maximize the full potential of VBN and promote its use, investigation of cases in which it is useful, determination of the optimum combination of procedures, a cost/benefit analysis and advancement of the VBN system are warranted.

(c) 2014 S. Karger AG, Basel

\section{Introduction}

With the widespread use of computed tomography (CT), peripheral pulmonary lesions (PPLs) have become common incidental findings in routine medical practice. More PPLs are detected on CT than on plain chest radiography, although many of the small lesions detected are benign [1]. It is therefore crucial to be able to distinguish lung cancer from benign lesions by using a safer and more accurate diagnostic method in order to avoid unnecessary surgical procedures. 
The three general management strategies for PPLs based on a patient's probability of malignancy, in order of low to high probability, are: (1) careful observation with serial CT scans, (2) tissue biopsy for pathologic diagnosis and (3) direct surgical excision. For PPLs with an indeterminate likelihood of malignancy, transthoracic needle aspiration (TTNA) or bronchoscopy is a less invasive diagnostic method than surgical biopsy [2]. One or other method is selected for individual patients on the basis of the clinical risks/benefits. TTNA is currently preferred, with a diagnostic yield of 90\% [95\% confidence interval (CI) 88-91\%], although this varies depending on if CT is used, the lesion size and whether the lesion is benign or malignant [3]. However, TTNA has a high incidence of complications, and the rates of development of pneumothorax and hemoptysis have been reported to be $15-20 \%$ and $1-5 \%$, respectively $[2,4]$. With bronchoscopy, the diagnostic yield of PPLs is insufficient, because the lesions are located beyond the segmental bronchus and are therefore not detectable. The American College of Chest Physicians (ACCP) guidelines reported that the sensitivities for PPLs larger and smaller than $2 \mathrm{~cm}$ were 63 and $34 \%$, respectively [3].

Presently, conventional bronchoscopy of PPLs is performed under X-ray fluoroscopy using a bronchoscope with an outer diameter of about 5-6 mm. Bronchoscopic diagnosis comprises 3 steps: (1) guiding a bronchoscope and biopsy instruments to the lesion, (2) confirmation of arrival at the lesion and (3) sampling from the lesion. Compared with central lesions, it is difficult to guide a bronchoscope and biopsy instruments to PPLs. To reach the PPLs, it is necessary to pass the bronchoscope and biopsy instruments through many bronchial bifurcations and to advance them with precision. To this end, bronchoscopists normally estimate the 3-dimensional distribution of the bronchus by referring to 2-dimensional planar axial slices on preexamination CT, and select a bronchial path during the examination. However, this method is inaccurate [5] and not applicable for bronchi that are more peripheral than the subsegmental bronchi.

To overcome these problems, navigational bronchoscopy has recently attracted considerable attention. Navigational bronchoscopy does not merely refer to various displayed CT data, but links these data and the actual patient information obtained in real time during bronchoscopy to guide the bronchoscope and biopsy instruments. Currently, virtual bronchoscopic navigation (VBN) [6] and electromagnetic navigation (EMN) [7] are used in clinical practice, and exclusive devices are sold for each system. These systems are similar with regard to the use of 3-dimensional CT display and virtual bronchoscopy; however, virtual bronchoscopic (VB) images are used in different ways, and EMN also makes use of an electromagnetic sensor [8].

Although several studies on VBN have been reported, these were mostly performed at a single expert center involving a small number of patients, and some were retrospective. The evidence level of each study was not very high. Recently, randomized comparative trials (RCTs) and meta-analysis studies with a high level of evidence have been reported.

In this review, we outline the features and aspects of VBN, review previous studies on this method (taking into consideration their evidence level), discuss the advantages and limitations of VBN and offer perspectives on how to maximize its application.

\section{Virtual Bronchoscopic Navigation}

Virtual bronchoscopy is a method for displaying 3-dimensional images of the tracheal and bronchial lumens prepared from the continuous volume data of helical CT as if they have been observed on bronchoscopy [9]. In $\mathrm{VBN}$, a bronchoscope is guided using VB images on the bronchial route to a PPL [6]. VBN can be performed using general-purpose software for VB image preparation. However, attention should be paid to the following points. VB images depend on the threshold values selected to differentiate between the airway wall and the lumen. In the peripheral airway, a bronchial bifurcation may be overlooked or a hole that appears to be a bifurcation may be presented in the bronchial wall, depending on the threshold setting. Therefore, an inappropriate threshold may guide the bronchoscope to an incorrect bronchus. To prevent this, it is necessary to set appropriate thresholds while confirming the presence or absence of a bifurcation in the axial, sagittal and coronal views so as to prepare accurate VB images [10]. Another important point is bronchoscope rotation on insertion. When a bronchoscope is rotated, the real bronchial image deviates from the VB image prepared earlier. The bronchoscope should therefore be advanced by rotating the VB image to adjust it to the real bronchial image at each bifurcation [11].

As a system exclusive to VBN for resolving these problems, Bf-NAVI ${ }^{\circledR}$ (Cybernet System Inc., Tokyo, Japan) was launched in 2008 in Japan, and LungPoint ${ }^{\circledR}$ (Broncus Medical Inc., Mountain View, Calif., USA) in 2009 in the USA. DirectPath ${ }^{\circledR}$ (Cybernet System Inc.) has recently replaced Bf-NAVI and is currently sold in China, where- 


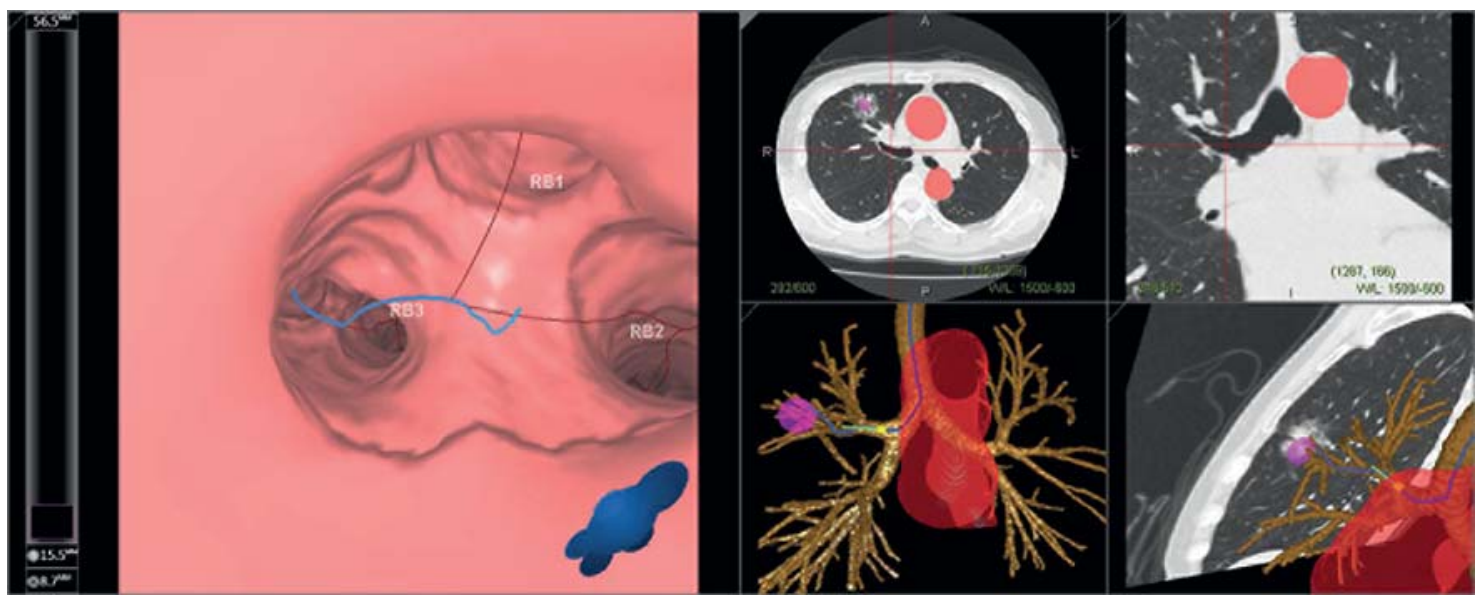

Fig. 1. LungPoint planning screen. Upper center: axial view. A PPL is shown in the right $S^{3}$ and a target is set on the lesion (pink circle). The red circle represents the aorta. Upper right: coronal view. Lower center: bronchial tree. The blue line on the bronchial tree is an automatically searched route to the lesion (pink circle). The yellow circle represents the tip of the virtual bronchoscope that moves in conjunction with VB images. The green line extending from the

as LungPoint is distributed in the USA, European Union and Japan. These VBN systems automatically search for a bronchial route to the target lesion by simply setting the target lesion, preparing VB images on the bronchial route and displaying the VB images in reference to the real images, although the functions do vary across devices.

\section{Techniques}

All VBN systems are composed of 2 phases: planning and guidance. Planning is a prebronchoscopy process to prepare for the production of VB images of a bronchial route to the target lesion. Guidance is the procedure to navigate a bronchoscope to the target lesion using VBN systems during bronchoscopy.

\section{Planning}

Firstly, CT scan and image reconstruction are performed under conditions recommended for each VBN system. The recommended slice thickness (and reconstruction interval) is $\leq 1 \mathrm{~mm}$. With thinner slices, VB images to more peripheral areas can be produced. When poor images are produced owing to respiratory artifacts, accurate VB images cannot be expected.

With LungPoint, automatic processing is initiated upon input of CT information in a DICOM (Digital Im- yellow circle represents the direction of the virtual bronchoscope, which is used for needle biopsy. The aorta is displayed in red. Lower right: composite display of the 3 cross-sectional CT images and bronchial tree. Left: VB image. The blue line on the image represents the bronchial route to the lesion. In this case, the VB image shows the right upper bronchus. The name of each bronchus in the upper lobe is displayed on the virtual images. aging and Communications in Medicine) format into the system, after which the airway is extracted. Axial, sagittal and coronal cross-sectional CT images are then displayed. When the target lesion is set in these CT images, 3 crosssectional images and the bronchial tree in which an automatically selected bronchial route to the target lesion are presented, and a VB image on the bronchial route is displayed. It is simple to operate, and a composite display of the 3 cross-sectional CT images and the bronchial tree is possible (fig. 1). The latest version, LungPoint 3.1, is applicable for fiducial marker placement for stereotactic body radiation therapy. It is also capable of presenting images as if they are observed under X-ray fluoroscopy as a virtual fluoroscopy view, and the target lesion and bronchial route can be presented in these images.

In the Japanese version of Bf-NAVI, when a target lesion is set, the automatically extracted bronchial lumen is presented in blue in 3 cross-sectional CT images, through which the bronchus extraction state can be confirmed. When a nonextractable bronchus is present, such as a peripheral bronchus near the lesion, it can be additionally extracted manually to prepare the VB image. Nonextracted bronchi are not visualized on VB images, and their bifurcations are also not displayed, which interferes with accurate navigation. Accordingly, confirmation of extraction of the bronchi and additional extraction are useful for increasing the navigation accuracy. 


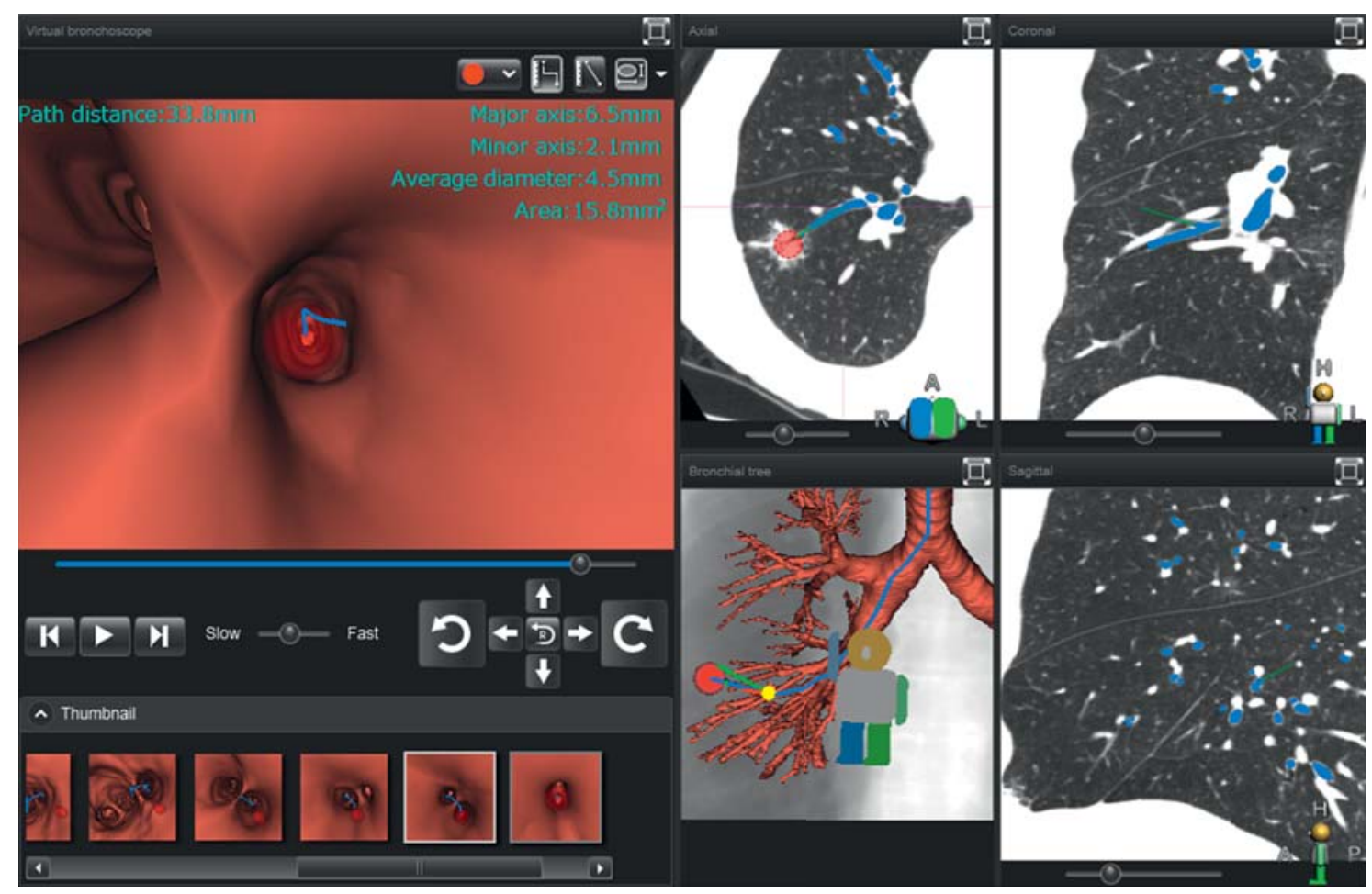

Fig. 2. DirectPath planning and guidance screen. Upper center: axial view. A PPL is shown in the right $S^{8}$ and a target is set on the lesion (red circle). The bronchus is automatically extracted by the system upon setting the target and is displayed in blue. A bronchus can be additionally extracted manually. Upper right: coronal view. Lower right: sagittal view. Lower center: bronchial tree. The blue line on the bronchial tree is an automatically searched route to the lesion (red circle). The yellow circle represents the tip of the virtual bronchoscope. The green line extending from the yellow circle represents the direction of the virtual bronchoscope. The back-

\section{Guidance}

The procedure performed during bronchoscopy is carried out to guide the bronchoscope to the target bronchus. Bf-NAVI presents the bronchus to which the bronchoscope is advanced on the VB image at each branching. The operator advances the bronchoscope manually by collating the VB image with the real image. By presenting VB images consistent with the real images on bronchoscopy, the position of the bronchoscope tip can be displayed on the CT images corresponding to the bronchial tree.

With the recently launched DirectPath (fig. 2), the bronchus extraction state can be confirmed in the axial, coronal and sagittal cross-sectional views during bronchoscopy when it is difficult to identify on the VB images whether a bronchial bifurcation is present or an artifact appears as a bifurcation. Additional extraction is also possible if necessary during bronchoscopy. ground is the virtual X-ray fluoroscopic image, which is linked with changes in the direction of the bronchial tree. Left: VB image. The blue line on the image represents the bronchial route to the lesion, and the red circle represents the target. The image on the bronchial route can be displayed corresponding to the real bronchial image. In this case, the VB image shows the 5th-generation bronchial bifurcation, and the blue line is shown heading toward the right $\mathrm{B}^{8}$ aiiay (6th-generation bronchus). Thumbnails of the bronchial bifurcations on the bronchial route are arranged under the VB image.
With LungPoint, the live bronchoscopic view is captured in the system, and the VB image that closely resembles the real image is automatically selected and displayed using image pattern recognition. At the same time, VB image information on the route and distance to the target lesion, the names of the bronchi and large vessels outside the bronchus are superimposed on the live bronchoscopic images and displayed (fig. 3). This system can automatically produce a VB image that is consistent with the real images, as described above. However, its image pattern recognition requires a large computer processing ability and a long time to select a VB image consistent with the real image [12]. Thus, the bronchoscope should be operated slowly. Moreover, when the bronchial lumen is not visible due to coughing, following the VB image is interrupted. 


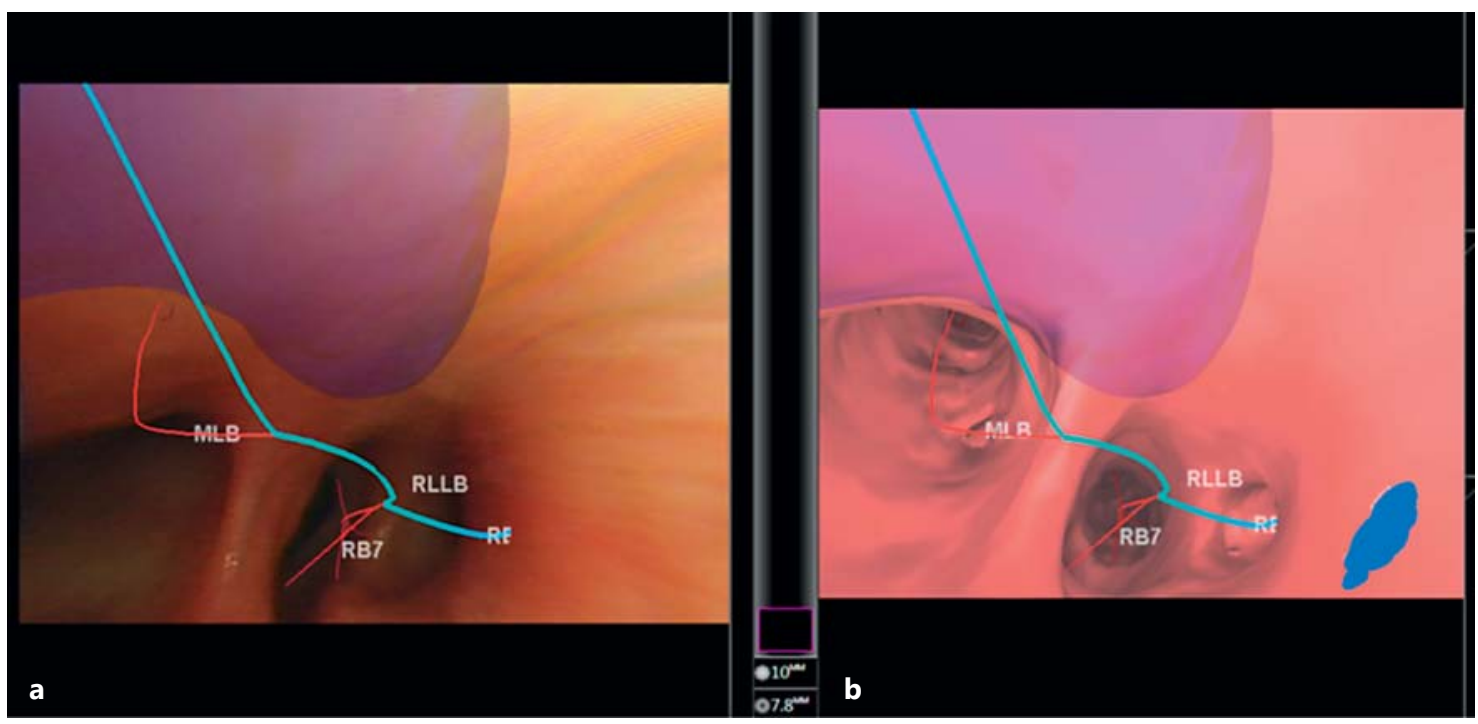

Fig. 3. LungPoint guidance screen. a Real bronchial image. b A VB image consistent with the real image is automatically selected and displayed. The bronchial route (blue line) to the lesion and the names of the bronchi are presented. These are also presented on the real image. Both images move in conjunction automatically.

\section{Clinical Applications}

\section{Diagnosis of PPLs}

In many cases, VBN is used in combination with CT, $\mathrm{X}$-ray fluoroscopy and endobronchial ultrasonograpy (EBUS) because the arrival of a biopsy instrument at the target lesion cannot be confirmed using VBN alone. A summary of the results of studies on the diagnosis of PPLs using VBN identified in a PubMed search as of November 2013, excluding reported studies involving fewer than 10 patients, is shown in table 1 . General anesthesia was performed in 1 study and the rest used conscious sedation. The study design, subjects and bronchoscope used varied among the studies. The results and characteristics by combining the confirmation methods are as follows.

\section{CT-Guided Ultrathin Bronchoscopy}

Ultrathin bronchoscopes with a working channel applicable for biopsy are now used not only to examine children but also to diagnose PPLs in adults $[13,14]$. Although there is no official definition of an ultrathin bronchoscope, generally, bronchoscopes with an outer diameter of about $\leq 3 \mathrm{~mm}$ are regarded as ultrathin in published reports. Ultrathin bronchoscopes can be inserted into a peripheral bronchus under direct vision, in contrast to conventional bronchoscopes, and they are useful for lesions difficult to diagnose by conventional bronchoscopy [15]. The diagnostic yield with a combination of VBN and CT-guided ultrathin bronchoscopy was reported to be within the range of $65.4-81.6 \%$, and that for lesions $\leq 2 \mathrm{~cm}$ was between 65.4 and $80.8 \%$ [11, 16, 17].

CT-guided ultrathin bronchoscopy may be useful for pure ground-glass opacity lesions, which are difficult to visualize using fluoroscopy and EBUS. On the other hand, there are problems with CT exposure during bronchoscopy and the cost of occupying a CT room. Thus, selection of patients is necessary. In addition, combination with cytology is necessary because only a small specimen can be collected using ultrathin bronchoscopy [15]. In ultrathin bronchoscopy, the diagnostic yield increases as the amount of collected specimen is increased through more frequent biopsy [18].

\section{EBUS with a Guide Sheath}

Radial-type (R)-EBUS is useful for identifying the localization of the lesion and sampling site in real time during the bronchoscopy of PPLs [19]. In fact, an increase in the diagnostic yield has been reported with R-EBUS [20]. On meta-analysis, the pooled sensitivity and specificity of R-EBUS for the detection of lung cancer in PPLs were 73\% (95\% CI 70-76\%) and 100\% (95\% CI 99-100\%), respectively [21]. Thus, EBUS is recommended in the ACCP guidelines [3]. Since EBUS is a method for confirming 
Table 1. Studies of VBN for the diagnosis of PPLs

\begin{tabular}{|c|c|c|c|c|c|c|c|c|c|c|c|c|c|}
\hline First author & Year & $\begin{array}{l}\text { Study } \\
\text { design }\end{array}$ & $\begin{array}{l}\text { VBN } \\
\text { system }\end{array}$ & $\begin{array}{l}\text { Bronchoscope } \\
\text { external } \\
\text { diameter }\end{array}$ & $\begin{array}{l}\text { Confir- } \\
\text { mation } \\
\text { of arrival }\end{array}$ & $\begin{array}{l}\text { Lesion } \\
\text { size } \\
\text { selection }\end{array}$ & $\begin{array}{l}\text { Le- } \\
\text { sions, } \\
\mathrm{n}\end{array}$ & $\begin{array}{l}\text { Diag- } \\
\text { nostic } \\
\text { yield }\end{array}$ & $\begin{array}{l}\text { Lesions } \\
<2 \mathrm{~cm}, \\
\mathrm{n}\end{array}$ & $\begin{array}{l}\text { Diagnostic } \\
\text { yield for } \\
\text { lesions } \\
<2 \mathrm{~cm}\end{array}$ & $\begin{array}{l}\text { Compli- } \\
\text { cations, } \\
\mathrm{n}\end{array}$ & $\begin{array}{l}\text { Compli- } \\
\text { cation } \\
\text { rate }\end{array}$ & Complications \\
\hline Shinagawa [16] & 2004 & Pro & Not used & $2.8 \mathrm{~mm}$ & CT & $<2 \mathrm{~cm}$ & 26 & $65.4 \%$ & 26 & $65.4 \%$ & 0 & $0.0 \%$ & None \\
\hline Asahina [26] & 2005 & Pro & Not used & 4.0 or $5.3 \mathrm{~mm}$ & EBUS & $\leq 3 \mathrm{~cm}$ & 30 & $63.3 \%$ & 18 & $44.4 \%$ & 0 & $0.0 \%$ & None \\
\hline Asano [11] & 2006 & Pro & Bf-NAVI & $2.8 \mathrm{~mm}$ & CT & $\leq 3 \mathrm{~cm}$ & 38 & $81.6 \%$ & 26 & $80.8 \%$ & $\mathrm{n} / \mathrm{a}$ & $\mathrm{n} / \mathrm{a}$ & $\mathrm{n} / \mathrm{a}$ \\
\hline Asano [10] & 2008 & Pro & Bf-NAVI & $4.0 \mathrm{~mm}$ & EBUS & $\mathrm{n} / \mathrm{a}$ & 32 & $84.4 \%$ & 15 & $73.3 \%$ & 0 & $0.0 \%$ & None \\
\hline Eberhardt [33] & 2010 & Pro & LungPoint & $2.8 \mathrm{~mm}$ & Non-Flu & $\mathrm{n} / \mathrm{a}$ & 25 & $80.0 \%$ & $\mathrm{n} / \mathrm{a}$ & $\mathrm{n} / \mathrm{a}$ & 1 & $4.0 \%$ & 1 PTX (0 CTI) \\
\hline Omiya [30] & 2010 & Retro & Bf-NAVI & 2.8 and $4.0 \mathrm{~mm}$ & Flu & $\leq 3 \mathrm{~cm}$ & 37 & $75.7 \%$ & 13 & $76.9 \%$ & $\mathrm{n} / \mathrm{a}$ & $\mathrm{n} / \mathrm{a}$ & $\mathrm{n} / \mathrm{a}$ \\
\hline Iwano [31] & 2011 & Retro & Not used & $2.8 \mathrm{~mm}$ & Flu & $\mathrm{n} / \mathrm{a}$ & 122 & $78.7 \%$ & 30 & $73.3 \%$ & $\mathrm{n} / \mathrm{a}$ & $\mathrm{n} / \mathrm{a}$ & $\mathrm{n} / \mathrm{a}$ \\
\hline Oshige [27] & 2011 & Pro & Bf-NAVI & 4.0 or $5.9 \mathrm{~mm}$ & EBUS & $\mathrm{n} / \mathrm{a}$ & 57 & $84.2 \%$ & 22 & $72.7 \%$ & 0 & $0.0 \%$ & None \\
\hline Ishida [24] & 2011 & $\mathrm{RCT}$ & Bf-NAVI & $4.0 \mathrm{~mm}$ & EBUS & $\leq 3 \mathrm{~cm}$ & 99 & $80.8 \%$ & 58 & $75.9 \%$ & 0 & $0.0 \%$ & None \\
\hline
\end{tabular}

Brad = Bradycardia CTI = chest tube insertion; Flu = fluoroscopy $;$ Hemo = hemorrhage; $/ \mathrm{a}=$ not available; Pro = prospective study; $\mathrm{PTX}=$ pneumothorax; Retro = retrospective study.

arrival at the target lesion and is not a navigation device, an unsuccessful visualization rate of $11-21 \%$ due to failure to reach the lesions was reported [22-24]. EBUS with a guide sheath (EBUS-GS) is a method in which arrival at the lesion is confirmed using an EBUS probe with a guide sheath; biopsy of the lesion is performed via a guide sheath placed in the lesion [25]. The diagnostic yield with a combination of VBN and EBUS-GS was found to be between 63.3 and $84.4 \%$, and that for lesions $\leq 2 \mathrm{~cm}$ between 44.4 and $75.9 \%$ [10, 24, 26-28]. The advantage of this method is that samples can be repeatedly collected accurately from the target lesion using EBUS-GS.

\section{X-Ray and Non-X-Ray Fluoroscopies}

The diagnostic yield with a combination of VBN and $\mathrm{X}$-ray fluoroscopy was reported to be between 62.5 and $78.7 \%$, and that for lesions $\leq 2 \mathrm{~cm}$ was between 54.5 and $76.9 \%$ [29-32]. Non-X-ray fluoroscopy-guided ultrathin bronchoscopy was shown in a previous study to have a high diagnostic yield of $80.0 \%$, although relatively large lesions observable by direct vision on ultrathin bronchoscopy were included [33].

\section{Summation}

Synthesizing the above studies, the overall diagnostic yield by VBN was $73.8 \%$ (95\% CI 70.9-76.8\%) and that for lesions $\leq 2 \mathrm{~cm}$ was $67.4 \%$ (95\% CI 63.3-71.5\%). Atten- tion should be paid to the fact that most studies were performed at a single expert center involving a small number of patients. Moreover, most studies using CT and EBUS also used X-ray fluoroscopy.

\section{RCTs and Meta-Analysis}

To demonstrate the usefulness of VBN systems objectively, 2 multicenter RCTs have been performed. Ishida et al. [24] randomly allocated 200 patients with PPLs ( $\leq 3$ $\mathrm{cm}$ ) to a VBN-assisted (VBNA) and a non-VBN-assisted (NVBNA) group, according to lesion size and the skill of the operators. A thin bronchoscope with an outer diameter of $4 \mathrm{~mm}$ was guided by a VBN system in the VBNA group and using axial CT images as a reference in the NVBNA group. Biopsy was performed concomitantly with EBUS-GS under X-ray fluoroscopy. The diagnostic yield in the VBNA group was $80.4 \%$, significantly higher than that $(67.0 \%)$ in the NVBNA group $(p=0.032)$. In addition, the time to initiation of the biopsy and the examination time were significantly shorter in the VBNA group. Asano et al. [32] randomly allocated 350 patients with PPLs $\leq 3 \mathrm{~cm}$ to 2 groups. An ultrathin bronchoscope with an outer diameter of $2.8 \mathrm{~mm}$ was guided with a VBN system in the VBNA group and using axial CT images as a reference in the NVBNA group. The ultrathin bronchoscope used had a thin working channel $(1.2 \mathrm{~mm})$ into which the EBUS probe could not be inserted. Thus, bi- 
opsy was performed under X-ray fluoroscopy in both groups. The diagnostic yield in the VBNA group was $67.1 \%$, higher than, but not significantly different from that $(59.9 \%)$ in the NVBNA group $(p=0.173)$. On subanalysis, the diagnostic yields of lesions located in the right upper lobe ( 81.3 vs. $53.2 \%, p=0.004)$, those located in the peripheral third of the lung field (64.7 vs. $52.1 \%$, $\mathrm{p}=0.047)$ and those invisible on posterior-anterior radiographs (63.2 vs. $40.5 \%, \mathrm{p}=0.043$ ) were significantly higher in the VBNA group. The respective reasons for the usefulness of VBN for these subgroups are as follows: (1) since the respiratory movement of the right upper lobe is small and there is almost no effect of the heartbeat, the preparation of virtual images and navigation of an ultrathin bronchoscope are simple, (2) more bronchial branchings are present toward the involved target for lesions located in the peripheral third than for those located in the central or intermediate third, but VBN enables selection of the correct bronchus at each branching and (3) a bronchoscope cannot be guided to lesions invisible on posterior-anterior radiography using fluoroscopy alone because such lesions are also mostly unidentifiable on fluoroscopy. There was no significant improvement of the diagnostic yield by VBN because unlike EBUS, confirmation of reaching the lesion using $\mathrm{X}$-ray fluoroscopy alone was insufficient and only a small amount of specimen could be biopsied using ultrathin bronchoscopy as only small biopsy forceps were applicable and the sampling frequency was insufficient.

The results common to the 2 RCTs were the ability to prepare VB images up to the 6th-generation bronchus (median) following the Japanese nomenclature [34], a high consistency between the virtual and real images (98 and $93.4 \%$, respectively) and bronchoscope insertion into more peripheral regions under direct vision in the VBNA group than in the NVBNA group, resulting in a higher arrival rate. In addition, there was no significant difference in the complication rates between the 2 groups in either RCT. On the other hand, no significant improvement of the diagnostic yield by VBN was demonstrated in the latter RCT as it was in the former. In the latter RCT, the presence of a group for which VBN was useful was assumed on subgroup analysis, and lesions belonging to this group accounted for $>70 \%$ of all the lesions. The results of the 2 RCTs show that VBN is useful for many patients but may not necessarily be required for all, and so selection of patients is necessary.

Wang Memoli et al. [35] performed a meta-analysis of 39 studies involving 3,004 patients with 3,052 lesions conducted from 2002 to 2010 to assess new techniques of im- age-guided bronchoscopy (i.e. EMN, VBN, R-EBUS, ultrathin bronchoscopy and/or the use of a guide sheath). They reported an overall pooled diagnostic yield of $70 \%$. The yield by VBN was $72.0 \%$ (95\% CI $65.7-78.4 \%$ ), although a study for mediastinal and hilar lesions, not for PPLs, was included.

\section{Factors Affecting the Diagnostic Yield}

On univariate analysis, lesion size $[26,29,31]$, the presence or absence of an involved bronchus [18], internal opacity $[28,31]$, position (distance from the chest wall) [31], bronchoscope insertion range [18], the number of samples collected [26] and examination time [26] were extracted in previous studies as factors that affect the diagnostic yield. The items investigated and the findings varied among the studies, however. On multivariate analysis, the location of lesions (the diagnostic yield was low in the left superior segment of the lower lobe) [18], the internal opacity of lesions (the yield for non-solid-type lesions was low) [31] and EBUS probe localization (the yield was high when the probe was within the lesion) [28] were established to be factors affecting the diagnostic yield. However, these factors should be carefully interpreted because the number of patients was insufficient in all studies, and the studies were markedly heterogeneous.

\section{Safety}

The complication rate in the reported studies ranged from 0 to $4 \%$. The overall complication rate was $1.0 \%$ (95\% CI 0.2-1.8\%). The complications that developed in 6 patients included pneumothorax $(\mathrm{n}=3)$, hemorrhage $(\mathrm{n}=2)$ and transient bradycardia $(\mathrm{n}=1)$. The complications were not severe and no complications were directly caused by VBN.

\section{Other Indications of $V B N$ \\ Marking of PPLs}

To identify the location of the lesion and the resection range in thoracoscopic surgery, Asano et al. [36] applied CT-guided transbronchial marking using VBN. The targets were 31 lesions that were $\leq 1 \mathrm{~cm}$ that displayed a pure ground-glass opacity pattern. No complications occurred in any patients, and the proximity of the lesions (the median distance from the lesion was $4 \mathrm{~mm}$, within $1 \mathrm{~cm}$ in 27 cases) could be marked with barium. The lesions could be resected with the barium mark en bloc in all cases of thoracoscopic partial resection. This method is applicable for multiple lesions because it causes no complications, unlike the percutaneous marking method which causes pneumothorax and hemorrhage. The procedure is sim- 
ple, so several marks can be applied to 1 lesion through which the resection range can be 3-dimensionally presented. Toba et al. [37] marked lesions with metallic coils using the same technique.

\section{Advantages of VBN}

TTNA is currently performed for PPLs. According to a cross-sectional analysis, the rate of complication by pneumothorax requiring drainage was $7 \%$ (95\% CI 6-7.2\%) and that including pneumothorax not requiring drainage was $15 \%$ (95\% CI 14-16\%), indicating a higher incidence. The incidence of hemorrhage was $1 \%$ (95\% CI $0.9-1.2 \%$ ) and blood transfusion was required in $18 \%$ [4] . Although the frequency of fatal complications like pleural dissemination and air embolism was low, these were also reported [38]. No complications directly caused by VBN were reported, and the VBN complication rate was equivalent to that on conventional bronchoscopy [39] and far lower than that on TTNA, which is a marked advantage. In addition, the $\mathrm{VBN}$-associated complication rate (1.0\%) appears to be lower than the EMN-associated complication rate (4\%) [40]. Although the reason for this is unclear, the following factors are worth considering: (1) with VBN, X-ray fluoroscopy was used in all but 1 study, (2) ultrathin bronchoscopy and exclusive thin biopsy instruments were used in many of the studies and (3) no needle was used, unlike those on EMN.

Another advantage of VBN is its high diagnostic yield, which was $67.4 \%$ for lesions $\leq 2 \mathrm{~cm}$. Although diagnostic yield cannot be simply compared - because it can be affected by not only the lesion size but also the target disease, location and presence or absence of an involved bronchus - the diagnostic yield of VBN is much higher than that of conventional bronchoscopy (34\%) reported in the ACCP guidelines [3]. The pooled sensitivity of TTNA for peripheral lung cancer was reported to be 90\%; however, analysis according to the lesion size was not performed [3]. It must be emphasized that a study with RCTs is necessary to compare the procedures. The applications of VBN are worth investigating, at least for nonoperable patients with lesions that are difficult to reach with TTNA and who are at high risk of pneumothorax [41].

Compared with EMN, VBN has the significant advantages of not requiring specific training because the technique is similar to conventional bronchoscopy and not needing a sensor or a specific biopsy instrument other than the system, thereby reducing costs [8]. Regarding the meta-analysis performed by Wang Memoli et al. [35], although many studies of EMN performed under general anesthesia were included with higher diagnostic yields under general anesthesia [40], the diagnostic yield with VBN (72.0\%) was still slightly higher than that with EMN (67.0\%). This was brought about by differences in the software used and the skill of the operators who prepared the VB images. However, the analyzed studies were performed only until 2010 and were all heterogeneous. In more recent studies of EMN, the diagnostic yield has improved [40]. Since both procedures have advantages as navigational bronchoscopy techniques, either procedure may be used depending on the characteristics of the lesions, the availability at institutions and the local expertise.

\section{Limitations of VBN}

\section{Reacquisition of CT Images}

The level of anatomical details visualized for virtual bronchoscopy depends on the acquired CT information. To prepare VB images of the route down to the peripheral bronchus, it is appropriate to minimize collimation $(0.5-1 \mathrm{~mm})$ and overlap the image reconstruction by at least $50 \%$ [42]. When VBN is planned from the beginning, it is important to store images reconstructed with slices having a thickness of $\leq 1 \mathrm{~mm}$ or raw CT data in order to be able to reconstruct images under the required conditions; this process prevents unnecessary exposure to repeat $\mathrm{CT}$ and reduces the cost.

\section{Discrepancy between Navigation Success and \\ Diagnostic Yield}

Ishida et al. [24] reported an arrival rate judged on EBUS images of $92.9 \%$, and the difference from the diagnostic yield was $12.1 \%$. Asano et al. [32], on the other hand, reported an arrival rate judged using X-ray imaging of $92.8 \%$, and the difference from the diagnostic yield was $25.7 \%$. One cause of the discrepancy between navigation success and diagnostic yield may be the accuracy of confirming the arrival, as the lesion may not actually be reached. Since CT information acquired before the examination is used for navigational bronchoscopy, navigation does not occur precisely in real time. Combination with a device capable of confirming the arrival and sampling site in real time (e.g. EBUS) is useful [43]. Another factor to consider is the relationship between the airway and the lesion. Similar to conventional bronchoscopy, it is difficult to collect specimens 
sufficiently from extrabronchial lesions, lesions that cannot be visualized by EBUS [25], lesions in which the involved bronchus cannot be confirmed on CT [18] and lesions not located in the linear direction from the involved bronchus even though the bronchus can be identified. For these lesions, an aspiration needle may be useful [44].

\section{Combination of the Use of a Bronchoscope,}

Reach-Confirming Method and Biopsy Method

Where the scope is inserted naturally depends on the outer diameter of the bronchoscope used. Although biopsy instruments cannot be guided to lesions using VBN, if a bronchoscope can be advanced to a more peripheral bronchus associated with the lesion using VBN, the distance between the bronchoscope and the lesion is shortened and it becomes unnecessary to guide biopsy instruments in many cases. On the other hand, the availability of diagnostic instruments (e.g. biopsy forceps, brush, needle, curette and guide sheath), the size of an insertable biopsy instrument and the amount of collectable tissue vary depending on the working channel diameter. Therefore, it is necessary to investigate the bronchoscope to be used and the method to confirm arrival as well as the biopsy method depending on the characteristics of the target lesion. Although the conditions were not directly compared but based on the results of 2 RCTs [24, 32], the ideal bronchoscope for carrying out VBN effectively may be a bronchoscope with a thin outer diameter insertable into peripheral bronchi and a working channel through which an EBUS radial probe (for accurate confirmation of arrival) and forceps (capable of collecting a sufficient amount of tissue) can be passed. Oki et al. [45] reported the diagnostic yield using a 3.4- $\mathrm{mm}$ bronchoscope with a 1.7-mm working channel. This bronchoscope may be useful because EBUS can be used, although a guide sheath cannot be applied.

\section{Future Prospects}

VBN is highly useful for guiding a bronchoscope to PPLs using VB images of the bronchial path. However, only limited expert institutions perform VBN. To achieve a more widespread use of VBN, several challenges must be overcome. Firstly, it is necessary to perform randomized comparative studies with TTNA and conventional bronchoscopy to clarify the position of VBN in the diagnostic methods for PPLs. Specifically, the type of lesions for which VBN is more useful than
TTNA and conventional bronchoscopy must be identified through these comparative studies which will determine the indication of VBN. Secondly, it is necessary to carry out a cost/benefit analysis. Thirdly, since the published results were mostly obtained from skilled operators, it is important to investigate the results in terms of the skill level of operators and the learning curve. Education programs and courses on VBN should be offered and performed. Fourthly, further improvement and advancement of the navigation system must be carried out. The VBN system would undoubtedly be easier to use if VB images more accurately reflected the real images.

Cryobiopsy has been attracting attention as a new biopsy method [46]. A recent RCT showed that the use of cryoprobes for diagnosing endobronchial malignancy was superior to forceps biopsy [47]. Cryoprobes have also been used to perform transbronchial biopsies for interstitial lung disease [48]. Schuhmann et al. [49] performed cryobiopsy combined with EBUS to diagnose PPLs, and could collect significantly larger samples without affecting safety compared with forceps biopsy. A thinner cryoprobe is currently under development [50]. Such an ultrathin cryoprobe may overcome the concerns regarding the small amount of collectable tissue using an ultrathin bronchoscope. In addition, an ultrathin scope in combination with a very thin ultrasound probe and an ultrathin cryoprobe may become a promising combination for VBN. Recently, Silvestri et al. [51] applied a new diagnostic method using a VBN system, namely bronchoscopic transparenchymal nodule access (BTPNA) to canines and achieved favorable outcomes. Using software exclusive for BTPNA (Archimedes, Broncus Medical Inc.), a route was planned for approaching the target lesion directly through the lung parenchyma avoiding blood vessels, and its point-of-entry was set in the central airway. Subsequently, a direct tunnel with a sheath reaching the lesion was bronchoscopically prepared along the planned route in the lung parenchyma by linking the VBN system and real fluoroscopic images for sample collection. BTPNA may be promising for lesions with no bronchus involvement or that may not be approachable bronchoscopically. However, investigation of BTPNA safety is equally necessary.

Taken together, the new procedures reported and analyzed here show the promising applications of VBN. Therefore, the use of VBN should be steadily promoted by identifying cases suitable for the procedure, carrying out a cost/benefit analysis and determining the optimum combination of procedures possible. 


\section{Acknowledgement}

The authors are indebted to Dr. Edward Barroga, Associate Professor and Senior Medical Editor of the Department of International Communications, Tokyo Medical University, for reviewing and editing the manuscript.

\section{Financial Disclosure and Conflicts of Interest}

F.A. has received speaker fees from Olympus Medical Systems Corporation as an invited guest speaker at academic medical meetings.

\section{References}

1 Aberle DR, Adams AM, Berg CD, Black WC, Clapp JD, Fagerstrom RM, Gareen IF, Gatsonis C, Marcus PM, Sicks JD: Reduced lung cancer mortality with low-dose computed tomographic screening. N Engl J Med 2011;365: 395-409.

$\checkmark 2$ Gould MK, Fletcher J, Iannettoni MD, Lynch WR, Midthun DE, Naidich DP, Ost DE: Evaluation of patients with pulmonary nodules: When is it lung cancer?: ACCP evidencebased clinical practice guidelines (2nd ed). Chest 2007;132:108S-130S.

3 Rivera MP, Mehta AC, Wahidi MM: Establishing the diagnosis of lung cancer: diagnosis and management of lung cancer, ed 3. American College of Chest Physicians evidencebased clinical practice guidelines. Chest 2013; 143:e142S-e165S.

- 4 Wiener RS, Schwartz LM, Woloshin S, Welch HG: Population-based risk for complications after transthoracic needle lung biopsy of a pulmonary nodule: an analysis of discharge records. Ann Intern Med 2011;155:137-144.

5 Dolina MY, Cornish DC, Merritt SA, Rai L, Mahraj R, Higgins WE, Bascom R: Interbronchoscopist variability in endobronchial path selection: a simulation study. Chest $2008 ; 133$ : 897-905.

-6 Asano F, Matsuno Y, Matsushita T, Seko A: Transbronchial diagnosis of a pulmonary peripheral small lesion using an ultrathin bronchoscope with virtual bronchoscopic navigation. J Bronchol 2002;9:108-111.

-7 Schwarz Y, Greif J, Becker HD, Ernst A, Mehta A: Real-time electromagnetic navigation bronchoscopy to peripheral lung lesions using overlaid CT images: the first human study. Chest 2006;129:988-994.

8 Leong S, Ju H, Marshall H, Bowman R, Yang I, Ree AM, Saxon C, Fong KM: Electromagnetic navigation bronchoscopy: a descriptive analysis. J Thorac Dis 2012;4:173-185.

-9 Vining DJ, Liu K, Choplin RH, Haponik EF: Virtual bronchoscopy. Relationships of virtual reality endobronchial simulations to actual bronchoscopic findings. Chest 1996;109: 549-553.

10 Asano F, Matsuno Y, Tsuzuku A, Anzai M, Shinagawa N, Yamazaki K, Ishida T, Moriya $\mathrm{H}$ : Diagnosis of peripheral pulmonary lesions using a bronchoscope insertion guidance system combined with endobronchial ultrasonography with a guide sheath. Lung Cancer 2008;60:366-373.
1 Asano F, Matsuno Y, Shinagawa N, Yamazaki K, Suzuki T, Ishida T, Moriya H: A virtual bronchoscopic navigation system for pulmonary peripheral lesions. Chest 2006;130:559566.

12 Mori K, Deguchi D, Sugiyama J, Suenaga Y, Toriwaki J, Maurer CR Jr, Takabatake H, Natori $\mathrm{H}$ : Tracking of a bronchoscope using epipolar geometry analysis and intensity-based image registration of real and virtual endoscopic images. Med Image Anal 2002;6:321336.

13 Rooney CP, Wolf K, McLennan G: Ultrathin bronchoscopy as an adjunct to standard bronchoscopy in the diagnosis of peripheral lung lesions. A preliminary report. Respiration 2002;69:63-68.

14 Saka H: Ultra-fine bronchoscopy: biopsy for peripheral lesions. Nippon Rinsho 2002; 60(suppl 5):188-190.

15 Matsuno Y, Asano F, Shindoh J, Abe T, Shiraki A, Ando M, Suzuki T, Seko A, Moriwaki $\mathrm{H}$ : Ct-guided ultrathin bronchoscopy: bioptic approach and factors in predicting diagnosis. Intern Med 2011;50:2143-2148.

16 Shinagawa N, Yamazaki K, Onodera Y, Miyasaka K, Kikuchi E, Dosaka-Akita H, Nishimura M: CT-guided transbronchial biopsy using an ultrathin bronchoscope with virtual bronchoscopic navigation. Chest 2004;125:11381143.

17 Shinagawa N, Yamazaki K, Onodera Y, Asano F, Ishida T, Moriya H, Nishimura M: Virtual bronchoscopic navigation system shortens the examination time - feasibility study of virtual bronchoscopic navigation system. Lung Cancer 2007;56:201-206.

-18 Shinagawa N, Yamazaki K, Onodera Y, Asahina H, Kikuchi E, Asano F, Miyasaka K, Nishimura M: Factors related to diagnostic sensitivity using an ultrathin bronchoscope under CT guidance. Chest 2007;131:549-553.

19 Herth FJ, Ernst A, Becker HD: Endobronchial ultrasound-guided transbronchial lung biopsy in solitary pulmonary nodules and peripheral lesions. Eur Respir J 2002;20:972974.

20 Paone G, Nicastri E, Lucantoni G, Dello Iacono R, Battistoni P, D’Angeli AL, Galluccio G: Endobronchial ultrasound-driven biopsy in the diagnosis of peripheral lung lesions. Chest 2005;128:3551-3557.
21 Steinfort DP, Khor YH, Manser RL, Irving LB: Radial probe endobronchial ultrasound for the diagnosis of peripheral lung cancer: systematic review and meta-analysis. Eur Respir J 2011;37:902-910.

22 Herth FJ, Eberhardt R, Becker HD, Ernst A: Endobronchial ultrasound-guided transbronchial lung biopsy in fluoroscopically invisible solitary pulmonary nodules: a prospective trial. Chest 2006;129:147-150.

-23 Kikuchi E, Yamazaki K, Sukoh N, Kikuchi J, Asahina H, Imura M, Onodera Y, Kurimoto N, Kinoshita I, Nishimura M: Endobronchial ultrasonography with guide-sheath for peripheral pulmonary lesions. Eur Respir $\mathrm{2004;}$ 24:533-537.

24 Ishida T, Asano F, Yamazaki K, Shinagawa N, Oizumi S, Moriya H, Munakata M, Nishimura M: Virtual bronchoscopic navigation combined with endobronchial ultrasound to diagnose small peripheral pulmonary lesions: a randomised trial. Thorax 2011;66:1072-1077.

25 Kurimoto N, Miyazawa T, Okimasa S, Maeda A, Oiwa H, Miyazu Y, Murayama M: Endobronchial ultrasonography using a guide sheath increases the ability to diagnose peripheral pulmonary lesions endoscopically. Chest 2004;126:959-965.

26 Asahina H, Yamazaki K, Onodera Y, Kikuchi E, Shinagawa N, Asano F, Nishimura M: Transbronchial biopsy using endobronchial ultrasonography with a guide sheath and virtual bronchoscopic navigation. Chest 2005; 128:1761-1765.

27 Oshige M, Shirakawa T, Nakamura M, Mineshita M, Kurimoto N, Miyazawa T, Becker HD: Clinical application of virtual bronchoscopic navigation system for peripheral lung lesions. J Bronchology Interv Pulmonol 2011; 18:196-202.

28 Tamiya M, Okamoto N, Sasada S, Shiroyama T, Morishita N, Suzuki H, Yoshida E, Hirashima T, Kawahara K, Kawase I: Diagnostic yield of combined bronchoscopy and endobronchial ultrasonography, under lungpoint guidance for small peripheral pulmonary lesions. Respirology 2013;18:834-839.

29 Tachihara M, Ishida T, Kanazawa K, Sugawara A, Watanabe $\mathrm{K}$, Uekita $\mathrm{K}$, Moriya $\mathrm{H}$, Yamazaki K, Asano F, Munakata M: A virtual bronchoscopic navigation system under Xray fluoroscopy for transbronchial diagnosis of small peripheral pulmonary lesions. Lung Cancer 2007;57:322-327. 
30 Omiya H, Kikuyama A, Kubo A, Okishio K, Kawaguchi T, Atagi S, Kawahara M, Takada M, Kusunoki Y: A feasibility and efficacy study on bronchoscopy with a virtual navigation system. J Bronchology Interv Pulmonol 2010;17:11-18.

- 31 Iwano S, Imaizumi K, Okada T, Hasegawa Y, Naganawa S: Virtual bronchoscopy-guided transbronchial biopsy for aiding the diagnosis of peripheral lung cancer. Eur J Radiol 2011; 79:155-159.

- 32 Asano F, Shinagawa N, Ishida T, Shindoh J, Anzai M, Tsuzuku A, Oizumi S, Morita S: Virtual bronchoscopic navigation combined with ultrathin bronchoscopy. A randomized clinical trial. Am J Respir Crit Care Med 2013; 188:327-333.

-33 Eberhardt R, Kahn N, Gompelmann D, Schumann M, Heussel CP, Herth FJ: LungPoint - a new approach to peripheral lesions. I Thorac Oncol 2010;5:1559-1563.

34 Fujisawa T, Tanaka M, Saka H: Report by the bronchus nomenclature working group. J Jpn Soc Bronchol 2000;22:330-331.

35 Wang Memoli JS, Nietert PJ, Silvestri GA: Meta-analysis of guided bronchoscopy for the evaluation of the pulmonary nodule. Chest 2012;142:385-393.

36 Asano F, Shindoh J, Shigemitsu K, Miya K, Abe T, Horiba M, Ishihara Y: Ultrathin bronchoscopic barium marking with virtual bronchoscopic navigation for fluoroscopy-assisted thoracoscopic surgery. Chest 2004;126:16871693.

- 37 Toba H, Kondo K, Miyoshi T, Kajiura K, Yoshida M, Kawakami Y, Takizawa H, Kenzaki K, Sakiyama S, Tangoku A: Fluoroscopy-assisted thoracoscopic resection after computed tomography-guided bronchoscopic metallic coil marking for small peripheral pulmonary lesions. Eur J Cardiothorac Surg 2013; 44:e126-e132.
8 Ibukuro K, Tanaka R, Takeguchi T, Fukuda $\mathrm{H}$, Abe S, Tobe K: Air embolism and needle track implantation complicating CT-guided percutaneous thoracic biopsy: single-institution experience. AJR Am J Roentgenol 2009; 193:W430-W436.

39 Du Rand IA, Blaikley J, Booton R, Chaudhuri N, Gupta V, Khalid S, Mandal S, Martin J, Mills J, Navani N, Rahman NM, Wrightson JM, Munavvar M: Summary of the British Thoracic Society guideline for diagnostic flexible bronchoscopy in adults. Thorax 2013;68: 786-787.

40 Gex G, Pralong JA, Combescure C, Seijo L, Rochat T, Soccal PM: Diagnostic yield and safety of electromagnetic navigation bronchoscopy for lung nodules: a systematic review and meta-analysis. Respiration 2014;87: 165-176.

41 Cox JE, Chiles C, McManus CM, Aquino SL, Choplin RH: Transthoracic needle aspiration biopsy: variables that affect risk of pneumothorax. Radiology 1999;212:165-168.

$\checkmark 42$ Neumann K, Winterer J, Kimmig M, Burger D, Einert A, Allmann KH, Hauer M, Langer M: Real-time interactive virtual endoscopy of the tracheo-bronchial system: influence of CT imaging protocols and observer ability. Eur J Radiol 2000;33:50-54.

43 Eberhardt R, Anantham D, Ernst A, FellerKopman D, Herth F: Multimodality bronchoscopic diagnosis of peripheral lung lesions: a randomized controlled trial. Am J Respir Crit Care Med 2007;176:36-41.
44 Chao TY, Chien MT, Lie CH, Chung YH, Wang JL, Lin MC: Endobronchial ultrasonography-guided transbronchial needle aspiration increases the diagnostic yield of peripheral pulmonary lesions: a randomized trial. Chest 2009;136:229-236.

45 Oki M, Saka H, Kitagawa C, Kogure Y, Murata N, Adachi T, Ando M: Randomized study of endobronchial ultrasound-guided transbronchial biopsy: thin bronchoscopic method versus guide sheath method. J Thorac Oncol 2012;7:535-541.

46 Hetzel J, Hetzel M, Hasel C, Moeller P, Babiak A: Old meets modern: the use of traditional cryoprobes in the age of molecular biology. Respiration 2008;76:193-197.

47 Hetzel J, Eberhardt R, Herth FJ, Petermann C, Reichle G, Freitag L, Dobbertin I, Franke KJ, Stanzel F, Beyer T, Moller P, Fritz P, Ott G, Schnabel PA, Kastendieck H, Lang W, MorresiHauf AT, Szyrach MN, Muche R, Shah PL, Babiak A, Hetzel M: Cryobiopsy increases the diagnostic yield of endobronchial biopsy: a multicentre trial. Eur Respir J 2012;39:685-690.

48 Babiak A, Hetzel J, Krishna G, Fritz P, Moeller P, Balli T, Hetzel M: Transbronchial cryobiopsy: a new tool for lung biopsies. Respiration 2009;78:203-208.

49 Schuhmann M, Bostanci K, Bugalho A, Warth A, Schnabel PA, Herth FJ, Eberhardt R: Endobronchial ultrasound-guided cryobiopsies in peripheral pulmonary lesions: a feasibility study. Eur Respir J 2014;43:233-239.

-50 Franke KJ, Nilius G, Ruehle KH, Enderle MD, Linzenbold W, von Weyhern CH: The cryoneedle: a new tool for histological biopsies. A feasibility study. Lung 2013;191:611-617.

-51 Silvestri GA, Herth FJ, Keast T, Rai L, Gibbs J, Wibowo H, Sterman DH: Feasibility and safety of bronchoscopictrans-parenchymal nodule access (BTPNA) in canines: a new realtime image-guided approach to lung lesions. Chest 2014;145:833-838. 